\title{
Parkinson's disease: Is there a light at the end of a tunnel?
}

\author{
Andrei Surguchov \\ VA Medical Center and Kansas University Medical Center, Kansas City, USA; asurguchov@kumc.edu
}

Received 22 August 2013; revised 22 September 2013; accepted 30 September 2013

Copyright (C) 2013 Andrei Surguchov. This is an open access article distributed under the Creative Commons Attribution License, which permits unrestricted use, distribution, and reproduction in any medium, provided the original work is properly cited.

Parkinson's disease is the second most common neurodegenerative disorder after Alzheimer disease affecting $1 \%-2 \%$ in people $>60$ years old and $3 \%-4 \%$ in people $>80$. It is a chronic, progressively disabling neurodegenerative disorder that occurs late in life, causing motor, cognitive, autonomic and emotional symptoms. There are four cardinal features of PD: tremor at rest, rigidity, akinesia (or bradykinesia) and postural instability [1]. The motor symptoms of Parkinson's disease result from the death of dopamine-generating cells in the substantia nigra, a region of the midbrain.

The cost of Parkinson's disease is substantial, including reduced quality of life, lost productivity, and increased health care expenditures. The number of persons with Parkinson's disease worldwide is expected to double by 2030 due to the aging of the population.

In the majority of cases $(\sim 95 \%)$, the disease occurs sporadically and is caused by the specific loss of dopaminergic neurons within the substantia nigra pars compacta. The identification of rare highly penetrant mutations in genes causing familial form of Parkinson's disease has had a considerable impact on our understanding of the pathogenesis of this complex and common disorder.

One of the hallmarks of the Parkinson's disease is the presence of Lewy bodies located basically in the substantia nigra. Lewy bodies are composed of different proteins including an aggregation prone protein $\alpha$-synuclein [2], and other proteins, e.g. synphililin-1, ubiquitin, neurofilaments and molecular chaperones. In addition to Lewy bodies, $\alpha$-synuclein in the brain of patients is present in the form of soluble oligomeric aggregates which possess toxic properties. Since Parkinson's disease is associated with the aberrant accumulation of $\alpha$-synuclein, it is referred to a group of synucleinopathies.

Our understanding of the genetic bases of Parkinson's disease has been enhanced by a number of large genome-wide association (GWA) studies which have collectively identified variants at over 18 loci that signifi- cantly increase the risk for this disorder.

At present, there is no cure for PD, but a variety of medications provide dramatic relief from the symptoms. Medical therapy for Parkinson's disease is most effective in treating the motor symptoms of resting tremor, bradykinesia, and rigidity. Levodopa remains the most effective current treatment for motor symptoms. Neurostimulation of the subthalamic nucleus is recommended for the management of motor fluctuations and dyskinesia in patients with advanced Parkinson's disease and severe motor complications.

Usually, patients are given levodopa combined with carbidopa. Carbidopa delays the conversion of levodopa into dopamine until it reaches the brain. Nerve cells can use levodopa to make dopamine and replenish the brain's dwindling supply. Although levodopa helps at least threequarters of parkinsonian cases, not all symptoms respond equally to the drug. Bradykinesia and rigidity respond best, while tremor may be only marginally diminished. Problems with balance and other symptoms may not be alleviated at all. Anticholinergics may help control tremor and rigidity. Other drugs, such as a potent agonist at dopamine D2 receptors bromocriptine, dopamine agonists pramipexole and ropinirole, mimic the role of dopamine in the brain, causing the neurons to react as they would to dopamine. An antiviral drug, amantadine increases dopamine release, blocks dopamine reuptake and also reduces symptoms. In May 2006, the FDA approved rasagiline to be used along with levodopa for patients with advanced PD or as a single-drug treatment for early PD.

Neurotrophic factors (also known as trophic or growth factors) are among the promising potential treatments to slow, stop or reverse Parkinson's disease in the brain. In pre-clinical models of Parkinson's disease, these proteins have been shown to promote the survival of dopamine neurons, as well as to induce these neurons' regrowth, which could have implications for slowing or stopping the progression of the disease rather than just temporarily 
masking symptoms. However, the use of neurotrophic factors is sometimes limited by the poor pharmacological properties, including the low blood-brain barrier permeability and relevant side effects. Development of small molecules with agonist activity, with better pharmacokinetics, and less side effects, is an attractive possibility.

The exciting news about the mechanism of Parkinson's disease comes from basic research concerning the structure and function of $\alpha$-synuclein. Growing evidence indicates a causative role of misfolded forms of the protein in this disorder, including the results of immunopathology and genetics. $\alpha$-Synuclein has long been defined as a" natively unfolded" monomer with molecular weight of about $14 \mathrm{kDa}$. A publication from Dennis Selkoe's group [3] states that $\alpha$-synuclein might exist in the brain, erythrocytes and other human cells as a folded tetramer of $58 \mathrm{kDa}$ caused a lot of discussions. Disputing the tetrameric $\alpha$-synuclein as a predominant form in the human tissue, the researchers from Thomas Südhof group have presented evidence that the brain $\alpha$-synuclein primarily consists of an unstructured monomer, but readily aggregates in a time-dependent manner [4].

Another interesting finding which might be important for better understanding of $\alpha$-synuclein role in pathogenesis of the Parkinson's disease is its prion-like properties. According to recent findings, misfolded $\alpha$-synuclein can induce Lewy-like pathology in cells that can spread from affected to unaffected region [5]

The significance of recent basic results in the field of Parkinson's disease and related neurodegenerative dis- eases gives us a hope that decades of discoveries pave the way for therapeutic breakthroughs. It is anticipated that latest findings will lead to new, efficacious treatments for the treatment of this devastating disorder.

\section{REFERENCES}

[1] Jankovic, J. (2008) Parkinson's disease: Clinical features and diagnosis. Journal of Neurology, Neurosurgery \& Psychiatry, 79, 368-376. http://dx.doi.org/10.1136/jnnp.2007.131045

[2] Surguchov, A. (2008) Molecular and cellular biology of synucleins. International Review of Cell and Molecular Biology, 270, 225-317. http://dx.doi.org/10.1016/S1937-6448(08)01406-8

[3] Bartels, T., Choi, J.G. and Selkoe, D.J. (2011) $\alpha$-Synuclein occurs physiologically as a helically folded tetramer that resists aggregation. Nature, 477, 107-110. http://dx.doi.org/10.1038/nature10324

[4] Burré, J., Vivona, S., Diao, J., Sharma, M., Brunger, A.T. and Südhof, T.C. (2013) Properties of native brain $\alpha$ synuclein. Nature, 498, E4-E6 http://dx.doi.org/10.1038/nature12125

[5] Olanow, C.W. and Brundin, P. (2013) Parkinson's disease and alpha synuclein: Is Parkinson's disease a prion-like disorder? Movement Disorders, 28, 31-40. http://dx.doi.org/10.1002/mds.25373 